\title{
Overexpression of mitofusin 2 improves translocation of glucose transporter 4 in skeletal muscle of high-fat diet-fed rats through AMP-activated protein kinase signaling
}

\author{
DEXIAN KONG ${ }^{1}$, GUANGYAO SONG ${ }^{1}, \mathrm{CHAO} \mathrm{WANG}^{2}$, HUIJUAN MA $^{2}$, \\ LUPING REN $^{2}$, QIAN NIE ${ }^{2}$, XUEMEI ZHANG ${ }^{2}$ and KEXIN GAN ${ }^{2}$ \\ ${ }^{1}$ Department of Internal Medicine, Hebei Medical University; \\ ${ }^{2}$ Department of Endocrinology, Hebei General Hospital, Shijiazhuang, Hebei 050017, P.R. China
}

Received January 29, 2013; Accepted April 23, 2013

DOI: $10.3892 / \mathrm{mmr} .2013 .1457$

\begin{abstract}
Mitofusin 2 (Mfn2) is a mitochondrial membrane protein that plays a role in mitochondrial fusion and metabolism in mammalian cells. Previous studies have reported a positive correlation between Mfn2 expression and insulin sensitivity in non-diabetic and type 2 diabetic subjects. Thus, the aim of the present study was to investigate whether Mfn2 overexpression improves insulin sensitivity of high-fat diet (HFD) rats and the possible underlying mechanisms. Male SD rats were randomly divided into four groups: negative control; HFD; HFD plus adenoviral vectors; and HFD plus adenoviral vectors encoding Mfn2. Following an 11-week treatment protocol, the euglycemic-hyperinsulinemic clamp technique was applied to evaluate insulin sensitivity in rats. The skeletal muscles from rats in each group were analyzed by real-time PCR and western blot analysis to determine glucose transporter 4 (GLUT4) expression, translocation and relative translocation signaling. Consistent with Mfn2 repression and glucose intolerance, HFD downregulates GLUT4 expression at the mRNA and protein levels, while Mfn2 overexpression activates AMP-activated protein kinase (AMPK), increases GLUT4 expression and translocation and improves insulin resistance in the skeletal muscles of HFD rats. Results of the present study indicate that Mfn2 overexpression improves insulin sensitivity and may regulate GLUT4 translocation in an AMPK-dependent manner in the skeletal muscles of HFD rats. This study is likely to provide insight into the unique role of $\mathrm{Mfn} 2$ in promoting glucose uptake, leading to modulation of GLUT4 translocation signaling and maintenance of glucose homeostasis in vivo.
\end{abstract}

Correspondence to: Dr Guangyao Song, Department of Internal Medicine, Hebei Medical University, 361 Zhongshan East Road, Shijiazhuang, Hebei 050017, P.R. China

E-mail: sguangyao2@163.com

Key words: mitofusin 2, glucose transporter 4, insulin resistance, AMP-activated protein kinase

\section{Introduction}

Mitochondrial dysfunction has been suggested to be involved in the development of insulin resistance (1). However, whether mitochondrial defects are the cause or consequence of insulin resistance remains controversial. One of the first studies connecting mitochondrial activity to diabetes mellitus was the identification of a mutation in the mitochondrial DNA that caused a maternal-inherited form of diabetes and associated deafness $(2,3)$. Studies in human subjects concerning this putative association indicated that the mitochondrial defect observed in diabetic muscle may be secondary to the insulin-resistant state instead of being a causal factor (4). In addition, functional defects of mitochondria, particularly reduced oxidative and phosphorylation capacities, which are secondary to insulin resistance, may aggravate insulin resistance.

Mitofusin 2 (Mfn2) is a mitochondrial membrane protein that plays a role in mitochondrial fusion and regulates mitochondrial metabolism in mammalian cells (5-7). It is highly expressed in a number of tissues, including skeletal muscle, heart and brain, with high energy demands (5). Mfn2 is relevant to numerous human diseases and is important in glucose metabolism and the development of insulin resistance. Mfn2 deficiency induces mitochondrial dysfunction, increases $\mathrm{H}_{2} \mathrm{O}_{2}$ concentration and activates c-Jun N-terminal kinase (JNK), leading to insulin resistance in skeletal muscle and liver (8). In addition, in myoblasts with a limited oxidative capacity, Mfn2 gain of function causes an increase in the glucose oxidation rate and a parallel increase in mitochondrial membrane potential, indicative of elevated pyruvate oxidation in mitochondria and enhanced Krebs cycle and oxidative phosphorylation (9).

Skeletal muscle glucose uptake and metabolism play a key role in the regulation of whole-body glucose homeostasis in normal and diabetic subjects $(10,11)$. Glucose uptake is the rate-limiting step of glucose utilization and is depressed in cases of insulin resistance, a process characterized by the reduced availability of sarcolemmal glucose transporter 4 (GLUT4) translocation and consequential lower glucose uptake $(12,13)$. GLUT4 is a glucose transport protein, highly expressed in adipose tissue and skeletal muscle, in which 
translocation is induced by two separate signal transduction pathways, an insulin-dependent and -independent pathway. The insulin-dependent pathway results in GLUT4 translocation via the activation of phosphatidylinositol-3 kinase (PI3K) and protein kinase $\mathrm{B} / \mathrm{Akt}$, whereas the AMP-activated protein kinase (AMPK) signaling pathway provides an alternative to the glucose uptake pathway in muscle. A defect in GLUT4 expression and translocation has been reported to be the primary metabolic abnormality in diabetic skeletal muscle (14). The aim of the present study was to examine the effects of Mfn2 on glucose homeostasis and correlative GLUT4 expression and translocation in high-fat diet (HFD) rats.

\section{Materials and methods}

Animals and experimental design. Forty male 4-week-old Sprague-Dawley rats (80-100 g) were obtained from the Animal Center of Hebei Medical University (Shijiazhuang, China) and maintained in an optimal environment for 1 week prior to experimentation. Animals were randomly divided into four groups: negative control $(\mathrm{NC} ; \mathrm{n}=10)$, high-fat diet (HF; $n=10)$, high-fat diet plus adenoviral vectors $(\mathrm{HF}+\mathrm{Ado}$; $\mathrm{n}=10)$ and high-fat diet plus adenoviral vectors encoding Mfn2 $(\mathrm{HF}+\mathrm{AdMfn} 2, \mathrm{n}=10)$. The rats were housed in a 12-h light-dark cycle and provided free access to a rodent standard diet $(65.5 \%$ carbohydrate, $10.3 \%$ fat and $24.2 \%$ protein) or a HFD $(20.1 \%$ carbohydrate, $59.8 \%$ fat and $20.1 \%$ protein), starting at 5 weeks of age, for 8 weeks. Following 8 weeks of HFD treatment, rats were subjected to a euglycemic-hyperinsulinemic clamp, as described previously, to assess insulin sensitivity (15).

Briefly, following collection of two basal samples, insulin was infused intravenously at a constant rate $(0.25 \mathrm{U} / \mathrm{kg} / \mathrm{h})$. Blood samples $(50 \mu \mathrm{l})$ were collected every $10 \mathrm{~min}$ to determine blood glucose and to adjust glucose infusion until the infusion rate was stabilized. Following 8 week HFD treatment, rats in the intervention groups $(\mathrm{HF}+\mathrm{Ado}$ and $\mathrm{HF}+\mathrm{AdMfn} 2)$ received $0.1 \mathrm{ml}$ Ado or AdMfn2 adenoviruses at a dose of $1 \times 10^{10}$ plaque-forming $\mathrm{U} / \mathrm{ml}$ via tail vein injection for 3 weeks and the non-intervention rats (NC and $\mathrm{HF}$ ) were injected with saline buffer only. At the end of the 11-week study, the rats were fasted overnight and the euglycemic-hyperinsulinemic clamp technique was applied to evaluate improvement in insulin resistance. Rats were anesthetized by intraperitoneal injections of pentobarbital sodium ( $50 \mathrm{mg} / \mathrm{kg}$ body weight) and blood samples were drawn from the abdominal aorta to measure the concentrations of glucose, insulin and free fatty acids (FFA) in plasma. Muscle tissues were collected, quickly placed in a liquid nitrogen container and stored at $-80^{\circ} \mathrm{C}$ for analysis. Experimental protocols were approved by the Animal Welfare Committee of the University and conducted in accordance with the institutional guidelines for animal research.

Quantitative real-time polymerase chain reaction ( $q P C R)$. Total RNA from rat muscle tissue was obtained using TRIzol reagent (Invitrogen Technologies, Carlsbad, CA, USA), according to the manufacturer's instructions. RNA concentration was determined by measuring the absorbance of a diluted sample at $260 \mathrm{~nm}$ in a UV spectrometer (ND 2000, Thermo, USA). Reverse transcription was performed on total RNA $(2 \mu \mathrm{g})$ using random primers to obtain the first-strand cDNA template. Real-time PCR was performed with $0.8 \mu \mathrm{l}$ cDNA (diluted 1:10), $2 \mu \mathrm{l}$ specific primers and 2X GoTaq ${ }^{\circledR}$ Green Master mix (Promega Corporation, Madison, WI, USA) in a final volume of $20 \mu \mathrm{l}$. PCR was performed as follows: an initial cycle at $95^{\circ} \mathrm{C}$ for $10 \mathrm{~min}$, followed by 40 cycles at $95^{\circ} \mathrm{C}$ for $15 \mathrm{sec}, 58^{\circ} \mathrm{C}$ for $20 \mathrm{sec}$ and $72^{\circ} \mathrm{C}$ for $27 \mathrm{sec}$. PCR products were analyzed by melting curve to confirm the specificity of amplification. Expression of Mfn2 and GLUT4 genes was analyzed and GAPDH was used as an internal control. The following sets of primers were used: Mfn2, 5'-AGCGTCCTCTCCCTCT GACA-3' and 5'-TTCCACACCACTCCTCCGAC-3'; GLUT4, 5'-CCCACAAGGCACCCTCACTA-3' and 5'-TGCCACCC ACAGAGAAGATG-3'; GAPDH, 5'-TGAACGGGAAGC TCACTG-3' and 5'-GCTTCACCACCTTCTTGATG-3'

Western blot analysis. Frozen-dried muscle tissues (50 mg) were homogenized in $500 \mu \mathrm{l}$ ice-cold homogenization buffer [1\% NP-40, $150 \mathrm{mmol} / 1 \mathrm{NaCl}, 50 \mathrm{mmol} / \mathrm{l}$ Tris ( $\mathrm{pH} \mathrm{8.0),} \mathrm{0.1 \%} \mathrm{apro-}$ tinin, $0.1 \%$ leupeptin, $0.035 \%$ pepstain A and $100 \mu \mathrm{g} / \mathrm{ml} \mathrm{PMSF]}$ for $30 \mathrm{~min}$ at $4^{\circ} \mathrm{C}$. The samples were subjected to centrifugation at $11,600 \mathrm{x} \mathrm{g}$ for $10 \mathrm{~min}$ at $4^{\circ} \mathrm{C}$. Protein concentrations of supernatants were determined using a BCA protein assay (Zomanbio, Beijing, China). Muscle protein fractions (30 $\mu \mathrm{g})$ were separated by $10 \%$ SDS-polyacrylamide gel electrophoresis and transferred to PVDF membranes (Millipore, USA). Following protein transfer, the membranes were blocked with $5 \%$ non-fat dry milk in TBST containing $0.05 \%$ Tween-20 overnight. Following blocking, membranes were incubated overnight with anti-IR $\beta$ (1:500), anti-AKT (1:800), anti-p-AKT (Ser-473; 1:200), anti-AMPK $\alpha$ (1:500), anti-p-AMPK $\alpha$ (Thr-172; 1:200), anti-Mfn2 (1:500) and anti-GLUT4 (1:500; All Bioworld Technology, Inc. Minneapolis, Mn, USA), followed by incubation with appropriate HRP-conjugated secondary antibodies (Santa Cruz Biotechnology, Santa Cruz, CA, USA) at room temperature for $1 \mathrm{~h}$. The membranes were then washed three times for $10 \mathrm{~min}$ in $0.05 \%$ Tween-20 in TBST. The immunoreactive proteins were visualized by enhanced chemiluminescence (Pierce Biotechnology, Rockford, IL, USA). $\mathrm{X}$-ray film was exposed to the PVDF membranes for $5 \mathrm{~min}$. The reaction product of each blot was analyzed by densitometry using the Bandscan 4.3 Software.

Membrane preparations of skeletal muscle. Following euglycemic hyperinsulinemic clamp experiments, muscle tissues were excised and immediately frozen in liquid nitrogen. Plasma membrane (PM) fractions of skeletal muscles were isolated from the muscles according to a method described previously (16).

Statistical analysis. Data were presented as the mean \pm SD and were analyzed using one-way analysis of variance followed by post hoc comparisons. $\mathrm{P}<0.05$ was considered to indicate a statistically significant difference.

\section{Results}

Plasma metabolic parameters. Following a total of 11-week-HFD treatment (shown in Table I), the HF group had higher plasma glucose, insulin and FFA levels than the $\mathrm{NC}$ group. In addition, plasma parameters following Ado or 
Table I. Plasma parameters at the end of the 11-week study.

\begin{tabular}{|c|c|c|c|c|}
\hline Plasma parameters & $\mathrm{NC}$ & $\mathrm{HF}$ & $\mathrm{HF}+$ Ado & $\mathrm{HF}+\mathrm{AdMfn} 2$ \\
\hline Glucose (mmol/l) & $6.00 \pm 0.64$ & $7.18 \pm 0.63^{\mathrm{a}}$ & $7.20 \pm 0.73^{\mathrm{a}}$ & $5.78 \pm 0.83^{b}$ \\
\hline Insulin (mU/l) & $28.88 \pm 4.81$ & $40.62 \pm 6.89^{\mathrm{a}}$ & $40.80 \pm 5.45^{\mathrm{a}}$ & $30.70 \pm 4.20^{\mathrm{b}}$ \\
\hline Fatty acids (mmol/l) & $0.64 \pm 0.09$ & $0.85 \pm 0.08^{a}$ & $0.82 \pm 0.04^{\mathrm{a}}$ & $0.65 \pm 0.08^{b}$ \\
\hline
\end{tabular}

Data are presented as the mean $\pm \mathrm{SD}\left(\mathrm{n}=5\right.$-8/group). ${ }^{\mathrm{a}} \mathrm{P}<0.05$, vs. $\mathrm{NC}$; and ${ }^{\mathrm{b}} \mathrm{P}<0.05$, vs. HF; one-way ANOVA. Mfn2, mitofusin 2 ; NC, negative control; HF, high-fat diet; Ado, adenoviral vector.

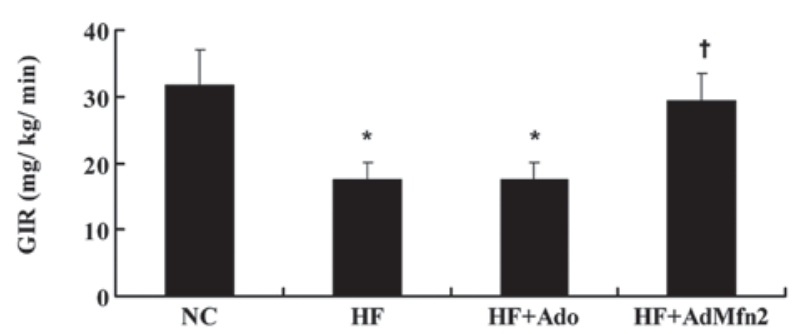

Figure 1. GIR during euglycemic-hyperinsulinemic clamp performed in NC, $\mathrm{HF}, \mathrm{HF}+$ Ado and HF + AdMfn2 groups. Data shown are the average of five separate experiments (mean $\pm \mathrm{SD}$ ). ${ }^{*} \mathrm{P}<0.05$, vs. $\mathrm{NC}$; ${ }^{\dagger} \mathrm{P}<0.05$, vs. HF; one-way ANOVA. GIR, glucose infusion rate; NC, negative control; $\mathrm{HF}$, high-fat diet; Ado, adenoviral vector.

AdMfn2 administration were analyzed in subsets of HFD rats. The parameters did not alter in the HF+Ado group compared with HF $(\mathrm{P}>0.05)$, but decreased in $\mathrm{HF}+\mathrm{AdMfn} 2$ compared with HF $(\mathrm{P}<0.05)$.

AdMfn2 increases the clamp glucose infusion rate (GIR). HFD caused a decrease in the clamp GIR required to maintain euglycemia (Fig. 1). AdMfn2 administration significantly increased GIR (61\%), whereas treatment of rats with Ado did not cause any alteration compared with the HF group.

$M f n 2$ is repressed in skeletal muscle in response to treatment with HFD and induced by administration of AdMfn2. To study the regulatory profile of Mfn2, its expression was analyzed under specific conditions, including exposure to HFD or treatment with AdMfn2, to stimulate whole-body Mfn2 expression. Exposure to HFD for 11 weeks caused downregulation of Mfn 2 mRNA and protein levels in skeletal muscles (Fig. 2; 51 and $44 \%$ of $\mathrm{NC}$, respectively; $\mathrm{P}<0.05)$, consistent with a previous study (1). Administration of AdMfn2 for three weeks led to a 3.4- and 3.3-fold increase in Mfn2 mRNA and protein expression in muscle tissues, respectively. In the $\mathrm{HF}+\mathrm{Ado}$ group, mRNA and protein levels were not altered in muscle tissue compared with the HF group.

AdMfn2 specifically induces GLUT4 $m R N A$ and protein expression in HFD rat muscle tissue. GLUT4 mediates glucose uptake in muscle tissue. To determine the role of the transporter in AdMfn2-induced insulin sensitivity improvement, GLUT4 expression levels were examined (Fig. 3). GLUT4 mRNA levels were measured by real-time PCR and normalized against the internal reference gene,
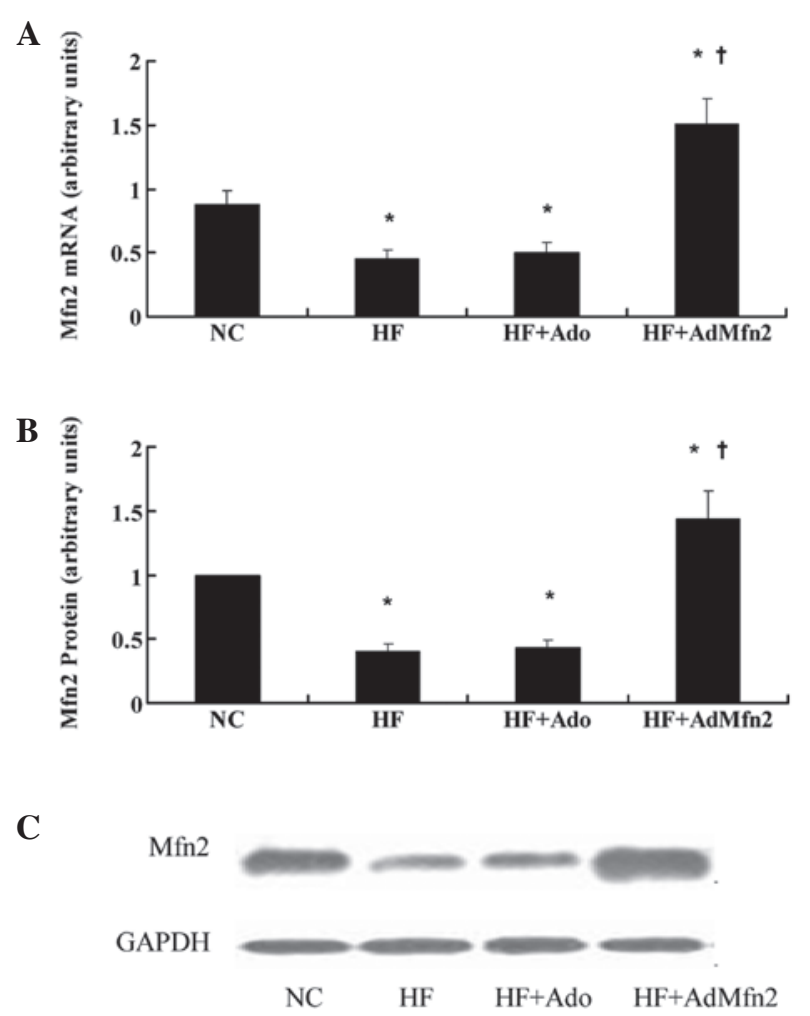

Figure 2. Mfn2 expression in skeletal muscles of $\mathrm{NC}, \mathrm{HF}, \mathrm{HF}+$ Ado and $\mathrm{HF}+$ AdMfn2 groups. (A) Mfn2 mRNA was quantified by real-time PCR. (B and C) Mfn2 protein was measured by western blot analysis. Data are shown as the average of three separate experiments performed in duplicate (mean $\pm \mathrm{SD}$ ). ${ }^{*} \mathrm{P}<0.05$, vs. $\mathrm{NC} ;{ }^{\dagger} \mathrm{P}<0.05$, vs. HF; one-way ANOVA. Mfn2, mitofusin 2; NC, negative control; HF, high-fat diet; Ado, adenoviral vector; PCR, polymerase chain reaction.

GAPDH. Results presented in Fig. 3A indicate a significant downregulation of GLUT4 mRNA by HFD (56\% of NC, $\mathrm{P}<0.05)$ and that Ado treatment did not have a significant effect on GLUT4 mRNA levels compared with the HF group. By contrast, following intervention of AdMfn2, GLUT4 mRNA was significantly higher in $\mathrm{HF}+\mathrm{AdMfn} 2$ compared with HF (149\% of HF; P<0.05; Fig. 3A). Consistent with previous observations (17), muscle expression of GLUT4 protein was markedly reduced in the $\mathrm{HF}$ group (66\% of $\mathrm{NC}$, $\mathrm{P}<0.05$; Fig. 3B). Following AdMfn2 treatment, total GLUT4 protein levels were significantly increased to $131 \%$ of the $\mathrm{HF}$ group ( $\mathrm{P}<0.05$; Fig. 3B). By contrast, there was no difference in total GLUT4 protein levels between the HF + Ado and HF groups ( $\mathrm{P}>0.05$; Fig. 3B). Therefore, administration of 
A

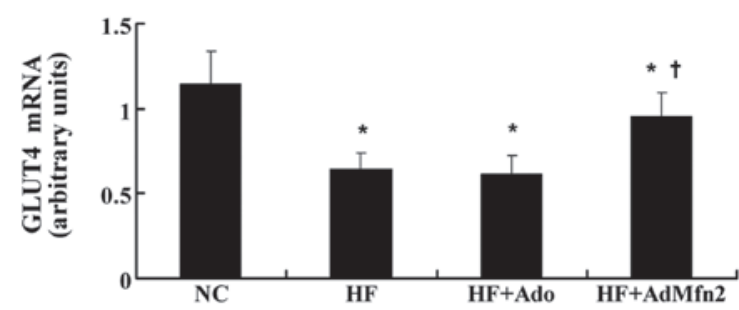

B

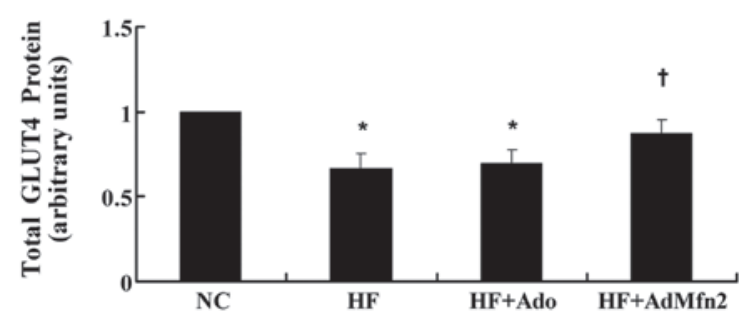

Figure 3. Effects of AdMfn2 on GLUT4 mRNA and total protein expression in rats muscle tissues from the $\mathrm{NC}, \mathrm{HF}, \mathrm{HF}+\mathrm{Ado}, \mathrm{HF}+\mathrm{AdMfn} 2$ groups. (A) Expression of GLUT4 mRNA was measured by real-time PCR. (B) Total GLUT4 protein was measured by western blot analysis. Data are shown as the average of three separate experiments performed in duplicate (mean $\pm \mathrm{SD}$ ). ${ }^{*} \mathrm{P}<0.05$, vs. NC; ${ }^{\dagger} \mathrm{P}<0.05$, vs. HF; one-way ANOVA. GLUT4, glucose transporter 4; Mfn2, mitofusin 2; NC, negative control; HF, high-fat diet; Ado, adenoviral vector; PCR, polymerase chain reaction.

\section{A}

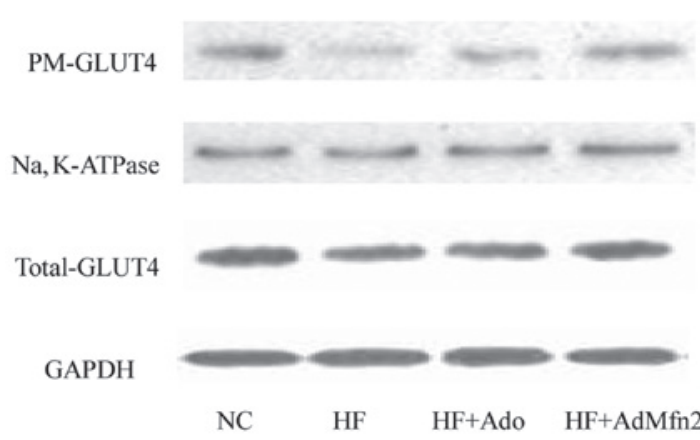

B

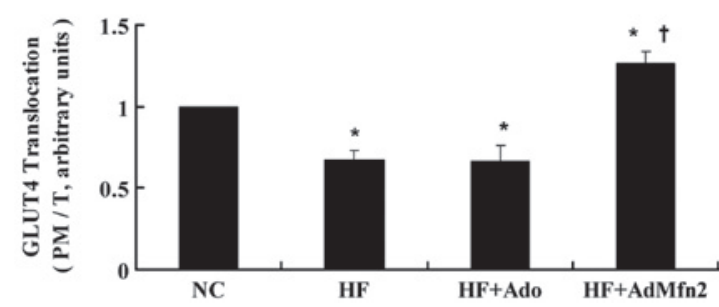

Figure 4. Effects of AdMfn2 on GLUT4 translocation in rat muscle tissues from the NC, HF, HF + Ado, HF + AdMfn2 groups. PM and total GLUT4 protein of muscle tissues was fractionated and subjected to western blot analysis. (A) Representative immunoblots are presented. (B) Ratio of PM to total GLUT4 protein is shown as mean \pm SD for three experiments. ${ }^{*} \mathrm{P}<0.05$, vs. NC; ${ }^{\dagger} \mathrm{P}<0.05$, vs. HF; one-way ANOVA. GLUT4, glucose transporter 4; Mfn2, mitofusin 2; NC, negative control; HF, high-fat diet; Ado, adenoviral vector.

AdMfn2 for 3 weeks enhanced GLUT4 gene expression and stabilized GLUT4 protein expression.

AdMfn 2 promotes GLUT4 translocation in HFD rat muscle tissues. To investigate the effect of AdMfn2 on GLUT4 translocation, the ratio of PM to total GLUT4 protein was analyzed
(Fig. 4). The PM and total GLUT4 protein of muscle tissues was fractionated and subjected to western blot analysis (Fig. 4A). Following administration of AdMfn2 to HFD rats, the ratio was significantly increased to $187 \%$ of the HF group $(\mathrm{P}<0.05$; Fig. 4B). However, the ratio was not significantly altered in the $\mathrm{HF}+$ Ado group compared with the HF group $(\mathrm{P}>0.05)$. These results demonstrate that AdMfn2 induces GLUT4 redistribution into the PM fraction in skeletal muscle tissue.

Role of Akt and AMPK pathways in AdMfn2-induced glucose uptake and GLUT4 translocation. To elucidate the effect of AdMfn2 on the signaling pathways involved in GLUT4 translocation, the IR $\beta$-PI3K-Akt and AMPK pathways were analyzed by western blot analysis (Fig. 5A). Results presented in Fig. 5B and $\mathrm{C}$ demonstrate that AdMfn2 had no effect on the phosphorylation of Akt ( $89 \%$ of HF; P $>0.05$ ) but significantly increased the phosphorylation of AMPK $\alpha$ (276\% of HF; $\mathrm{P}<0.05)$. No differences were detected with regard to the expression of AMPK $\alpha(\mathrm{P}>0.05$; Fig. $5 \mathrm{C})$. Expression of IR $\beta$, PI-3K, p-AKT and AKT protein was significantly decreased in the HF group compared with $\mathrm{NC}(\mathrm{P}<0.05$; Fig. $5 \mathrm{~B}$ and $\mathrm{D})$. No significant differences were found in the expression levels of IR $\beta, \mathrm{PI}-3 \mathrm{~K}, \mathrm{p}-\mathrm{AKT}$ and AKT protein among the three groups (HF, HF + Ado and HF + AdMfn2; P>0.05; Fig. 5B and D). These results demonstrate that the effect of AdMfn2 on GLUT4 translocation contributes, in part, to increased AMPK activation.

\section{Discussion}

Results of the present study indicate that Mfn2 improves insulin sensitivity and may regulate GLUT4 translocation in an AMPK-dependent manner in skeletal muscles of HFD rats. In the present study, HFD treatment in rats was found to induce insulin resistance and reduce Mfn2 expression in insulin-resistant rats. These results are in agreement with the previous observations that muscle Mfn2 is repressed in Zucker rats and diabetes patients $(1,18,19)$.

Previous studies have indicated that Mfn2 plays a positive role in maintaining glucose homeostasis. In addition, aberrant expression of Mfn2 may be involved in the pathophysiology of insulin resistance. Therefore, consistent with Mfn2/shRNA $\mathrm{BALB} / \mathrm{c}$ mice, hepatic glucose production and insulin resistance was significantly increased (20). An additional study has also reported that Mfn2 deficiency causes mitochondrial dysfunction, leading to enhanced reactive oxygen species production and JNK activity and inactivation of insulin receptor substrate 1, a key protein in insulin signaling (8). A positive correlation between Mfn2 expression and insulin sensitivity was also previously detected in non-diabetic and type 2 diabetic subjects (1). Consistent with these observations a $67 \%$ increase in whole-body insulin sensitivity was noted in the current study, following administration of AdMfn2 for three weeks in HFD rats (as indicated by clamp GIR). However, the mechanism by which Mfn2 overexpression ameliorates GIR must be further investigated.

HFD rats demonstrated glucose intolerance, which is, at least in part, attributable to decreased glucose uptake. Glucose transport mediated by GLUT4 is a major rate-limiting step in glucose metabolism in skeletal muscle, which may be activated 
A

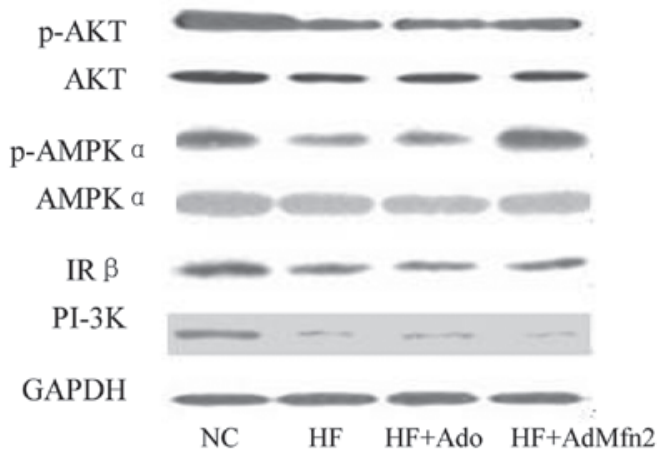

B

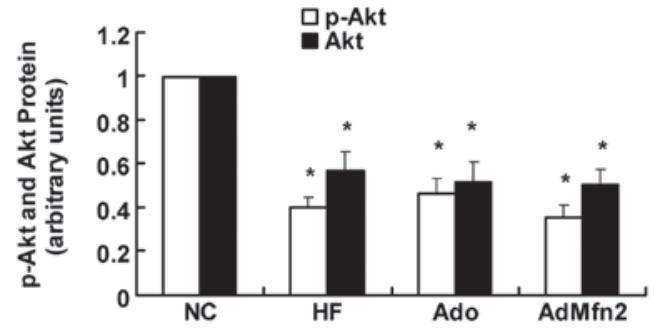

C

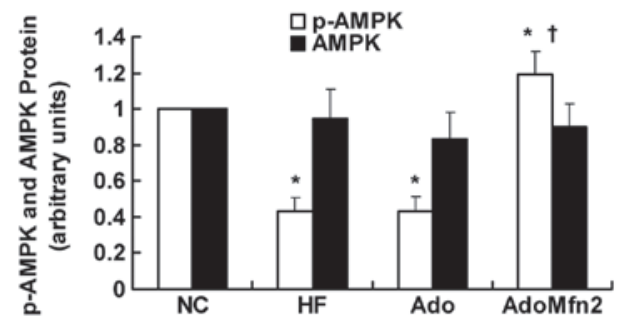

D

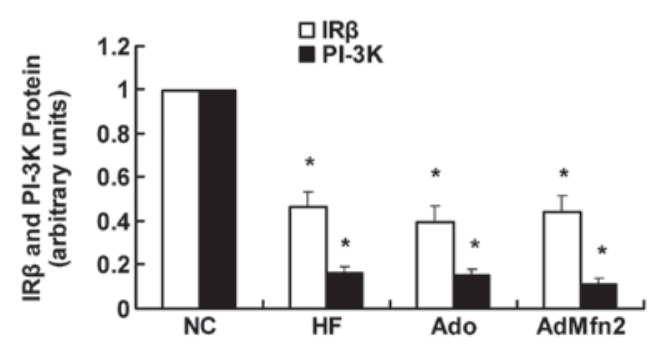

Figure 5. Effects of AdMfn2 on the signaling pathways involved in GLUT4 translocation in rat muscle tissues from the NC, HF, HF + Ado and HF + AdMfn2 groups. (A) Expression of phosphorylated Akt (Ser473), Akt, phosphorylated AMPK (Thr172), AMPK, IR $\beta$ and PI3K were measured by western blot analysis. (B-D) Phosphorylated Akt, Akt, phosphorylated AMPK, AMPK, IR $\beta$ and PI-3K protein levels were calculated as ratios. Data are shown as the average of three separate experiments performed in duplicate (mean $\pm \mathrm{SD}$ ). ${ }^{*} \mathrm{P}<0.05$, vs. $\mathrm{NC} ;{ }^{\dagger} \mathrm{P}<0.05$, vs. HF; one-way ANOVA. GLUT4, glucose transporter 4; AMPK, AMP-activated protein kinase; IR $\beta$, insulin receptor $\beta$; PI3K, phosphatidylionositol-3 kinase; NC, negative control; HF, high-fat diet; Ado, adenoviral vector.

by two separate signaling pathways. Impaired glucose transport in skeletal muscle leads to impaired whole body glucose uptake and contributes to the pathogenesis of type 2 diabetes mellitus. Previous studies have reported whole-body glucose uptake as a linear function of GLUT4 expression in skeletal muscle (21). A previous study of six morbidly obese females who underwent malabsorptive bariatric surgery, revealed an increase in Mfn2 mRNA levels, associated with the improvement of whole-body glucose uptake, as well as with an increase in GLUT4 expression (19). In the present study, Mfn2 overexpression increased GLUT4 expression and translocation in skeletal muscles of HFD rats, which coincided with an increase in AMPK phosphorylation rather than Akt phosphorylation. A number of previous studies have described an association between Mfn2 expression and Akt phosphorylation $(8,22-25)$. Current results demonstrate that overexpression of $\mathrm{Mfn} 2$ has no effect on Akt activation in skeletal muscles of HFD rats, consistent with previous observations indicating that upregulated Mfn2 does not affect Akt phosphorylation in cultured cardiomyocytes (22). However, other studies have reported Mfn2 overexpression depresses the activation of Akt or, Mfn2 knockdown attenuated the activation of Akt (8,23-25). Thus, the correlation between Mfn2 expression and Akt phosphorylation remains poorly understood due to signaling discrepancies and cell selection.

Physiological and pathophysiological conditions characterized by altered glucose utilization (diabetes, obesity, insulin resistance, exercise and weight loss) leads to changes in Mfn2 expression. However, direct evidence of the association of Mfn2 in glucose uptake and insulin sensitivity has not been reported. In the present study, a positive correlation between Mfn2 expression and insulin sensitivity assessed by clamp GIR was reported, consistent with previous studies $(1,19)$. In addition, GLUT4 mRNA levels have been found to positively correlate with Mfn2 mRNA concentrations and linearly correlate with whole-body glucose uptake (19). However, the direct correlation between Mfn2 and GLUT4 remains unclear. In the present study, it was demonstrated that Mfn2 overexpression induced GLUT4 expression and translocation via AMPK activation in skeletal muscles of HFD rats, which was important for improving insulin sensitivity.

Based on the role of Mfn2 in improvement of glucose uptake, one of the limitations of this study was that the hypothesis of a potential signaling pathway by which Mfn2 overexpression caused increased GLUT4 translocation was made, however, we did not reach a final conclusion as to whether the increase of GLUT4 translocation correlates with AMPK phosphorylation via Mfn2 upregulation in muscle tissues. In addition, a specific mechanism by which Mfn2 regulates GLUT4 expression by AMPK phosphorylation in skeletal muscles has yet to be established. Thus, future studies are required to establish the specific cause-and-effect correlation between Mfn2 expression and AMPK phosphorylation and to identify the detailed signaling pathways by which Mfn2 regulates GLUT4 expression.

In summary, results of the current study indicate that insulin resistance is associated, at baseline, with a reduced expression of Mfn2 in skeletal muscle, which is associated with a concomitant reduction in expression and translocation activity of GLUT4. In addition, Mfn2 overexpression increased glucose uptake by enhanced GLUT4 translocation, which was induced via increased AMPK phosphorylation. Increase in Mfn2 expression and the concomitant stimulation of AMPK activity may be relevant to enhanced glucose uptake capacity in insulin-resistant rats.

\section{Acknowledgements}

This study was supported by grants from the National Natural Science Foundations of China (nos. 30971391 and 
81170742) and Hebei Natural Science Foundation of China (nos. C2010001638 and C2011307008).

\section{References}

1. Bach D, Naon D, Pich S, et al: Expression of Mfn2, the Charcot-Marie-Tooth neuropathy type 2A gene, in human skeletal muscle: effects of type 2 diabetes, obesity, weight loss and the regulatory role of tumor necrosis factor alpha and interleukin-6. Diabetes 54: 2685-2693, 2005.

2. van den Ouweland JM, Lemkes HH, Ruitenbeek W, et al: Mutation in mitochondrial tRNA(Leu)(UUR) gene in a large pedigree with maternally transmitted type II diabetes mellitus and deafness. Nat Genet 1: 368-371, 1992.

3. Zorzano A, Liesa M and Palacin M: Mitochondrial dynamics as a bridge between mitochondrial dysfunction and insulin resistance. Arch Physiol Biochem 115: 1-12, 2009.

4. Hoeks J and Schrauwen P: Muscle mitochondria and insulin resistance: a human perspective. Trends Endocrinol Metab 23: 444-450, 2012.

5. Bach D, Pich S, Soriano FX, et al: Mitofusin-2 determines mitochondrial network architecture and mitochondrial metabolism A novel regulatory mechanism altered in obesity. J Biol Chem 278: 17190-17197, 2003.

6. Chen H, Detmer SA, Ewald AJ, Griffin EE, Fraser SE and Chan DC: Mitofusins Mfn1 and Mfn2 coordinately regulate mitochondrial fusion and are essential for embryonic development. J Cell Biol 160: 189-200, 2003.

7. Soriano FX, Liesa M, Bach D, Chan DC, Palacin M and Zorzano A: Evidence for a mitochondrial regulatory pathway defined by peroxisome proliferator-activated receptor-gamma coactivator-1 alpha, estrogen-related receptor-alpha and mitofusin 2. Diabetes 55: 1783-1791, 2006.

8. Sebastián D, Hernández-Alvarez MI, Segalés J, et al: Mitofusin 2 (Mfn2) links mitochondrial and endoplasmic reticulum function with insulin signaling and is essential for normal glucose homeostasis. Proc Natl Acad Sci USA 109: 5523-5528, 2012.

9. Pich S, Bach D, Briones P, et al: The Charcot-Marie-Tooth type 2A gene product, Mfn2, up-regulates fuel oxidation through expression of OXPHOS system. Hum Mol Genet 14: 1405-1415, 2005.

10. McConell GK and Wadley GD: Potential role of nitric oxide in contraction-stimulated glucose uptake and mitochondrial biogenesis in skeletal muscle. Clin Exp Pharmacol Physiol 35: 1488-1492, 2008.

11. Zhao HL, Liu LZ, Sui Y, et al: Fatty acids inhibit insulin-mediated glucose transport associated with actin remodeling in rat L6 muscle cells. Acta Diabetol 47: 331-339, 2010.

12. Jaiswal N, Yadav PP, Maurya R, Srivastava AK and Tamrakar AK: Karanjin from Pongamia pinnata induces GLUT4 translocation in skeletal muscle cells in a phosphatidylinositol-3-kinase-independent manner. Eur J Pharmacol 670: 22-28, 2011.

13. Kim JY, Jo KJ, Kim BJ, Baik HW and Lee SK: $17 \beta$-estradiol induces an interaction between adenosine monophosphateactivated protein kinase and the insulin signaling pathway in 3T3-L1 adipocytes. Int J Mol Med 30: 979-985, 2012.
14. Bi Y, Sun WP, Chen X, Li M, Liang H, Cai MY, Zhu YH, He XY, $\mathrm{Xu} F$ and Weng JP: Effect of early insulin therapy on nuclear factor kappaB and cytokine gene expressions in the liver and skeletal muscle of high-fat diet, streptozotocin-treated diabetic rats. Acta Diabetol 45: 167-178, 2008.

15. Iglesias MA, Ye JM, Frangioudakis G, et al: AICAR administration causes an apparent enhancement of muscle and liver insulin action in insulin-resistant high-fat-fed rats. Diabetes 51: 2886-2894, 2002.

16. Sato T, Man ZW, Toide K and Asahi Y: Plasma membrane content of insulin-regulated glucose transporter in skeletal muscle of the male Otsuka Long-Evans Tokushima Fatty rat, a model of non-insulin-dependent diabetes mellitus. FEBS Lett 407: 329-332, 1997.

17. Wang ZQ, Zhang XH, Yu Y, et al: Bioactives from bitter melon enhance insulin signaling and modulate acyl carnitine content in skeletal muscle in high-fat diet-fed mice. J Nutr Biochem 22: 1064-1073, 2011.

18. Hernández-Alvarez MI, Thabit H, Burns N, et al: Subjects with early-onset type 2 diabetes show defective activation of the skeletal muscle PGC-1\{alpha\}/Mitofusin-2 regulatory pathway in response to physical activity. Diabetes Care 33: 645-651, 2010.

19. Mingrone G, Manco M, Calvani M, Castagneto M, Naon D and Zorzano A: Could the low level of expression of the gene encoding skeletal muscle mitofusin-2 account for the metabolic inflexibility of obesity? Diabetologia 48: 2108-2114, 2005.

20. Chen X and Xu Y: Liver-specific reduction of Mfn2 protein by RNAi results in impaired glycometabolism and lipid homeostasis in BALB/c mice. J Huazhong Univ Sci Technolog Med Sci 29: 689-696, 2009.

21. Mingrone G, Rosa G, Di Rocco P, et al: Skeletal muscle triglycerides lowering is associated with net improvement of insulin sensitivity, TNF-alpha reduction and GLUT4 expression enhancement. Int J Obes Relat Metab Disord 26: 1165-1172, 2002.

22. Yu H, Guo Y, Mi L, Wang X, Li L and Gao W: Mitofusin 2 inhibits angiotensin II-induced myocardial hypertrophy. J Cardiovasc Pharmacol Ther 16: 205-211, 2011.

23. Guo YH, Chen K, Gao W, et al: Overexpression of Mitofusin 2 inhibited oxidized low-density lipoprotein induced vascular smooth muscle cell proliferation and reduced atherosclerotic lesion formation in rabbit. Biochem Biophys Res Commun 363: 411-417, 2007.

24. Lugus JJ, Ngoh GA, Bachschmid MM and Walsh K: Mitofusins are required for angiogenic function and modulate different signaling pathways in cultured endothelial cells. J Mol Cell Cardiol 51: 885-893, 2011.

25. Wan-Xin T, Tian-Lei C, Ben W, Wei-Hua W and Ping F: Effect of mitofusin 2 overexpression on the proliferation and apoptosis of high-glucose-induced rat glomerular mesangial cells. J Nephrol 25: 1023-1030, 2012. 